\title{
Verapamil responsive incessant ventricular tachycardia resulting in severe ventricular dysfunction in a young child: successful management with oral verapamil
}

\author{
Jae-Sook Ma, Byung-Ju Kim, Jeong-Gwan Cho
}

\author{
Department of \\ Paediatrics, Chonnam \\ University Medical \\ School, Kwangju, \\ Korea \\ J S Ma \\ B J Kim \\ Department of \\ Internal Medicine, \\ Division of Cardiology, \\ Chonnam University \\ Medical School, \\ Kwangju, Korea \\ J G Cho \\ Correspondence to: \\ Dr Jae-Sook Ma, \\ Department of Paediatrics, \\ Chonnam University \\ Medical School, \\ 8 Hak-Dong, Tong-Ku, \\ Kwangju 501-757, Korea. \\ Accepted for publication \\ 29 October 1996
}

\begin{abstract}
In young children with incessant ventricular tachycardia and severe ventricular dysfunction, the management of tachycardia with conventional antiarrhythmic drugs remains a major therapeutic challenge because most of these drugs can further depress myocardial function. We report a four year old boy with verapamil responsive incessant ventricular tachycardia and severe ventricular dysfunction in whom oral verapamil treatment eliminated both the arrhythmia and the picture of dilated cardiomyopathy. On oral verapamil, the patient remains asymptomatic without recurrence of the ventricular tachycardia over a follow up period of 10 months.
\end{abstract}

(Heart 1997;77:286-287)

Keywords: verapamil responsive ventricular tachycardia; tachycardia induced cardiomyopathy; verapamil; child
Idiopathic verapamil responsive left ventricu- $\vec{\omega}$ lar tachycardia with a QRS pattern of right bundle branch block and left axis deviation has been well described as a distinct arrhyth- ㄱ mia entity in patients without apparent heart $\omega$ disease. ${ }^{12}$ This variety of ventricular tachycardia is usually well tolerated and is not often o associated with tachycardia induced cardiomyopathy. To the best of our knowledge, it $\bar{z}$ has rarely been reported in patients younger than 10 years. There are no published data $\stackrel{\Im}{\supset}$ concerning the acute and chronic efficacy of $\vec{\theta}$ oral verapamil in very young children with this unique type of arrhythmia associated with i tachycardia induced cardiomyopathy.

We describe here a four year old boy with verapamil responsive incessant ventricular tachycardia and severe ventricular dysfunction $\stackrel{\odot}{\varnothing}$ in whom oral verapamil treatment eliminated both the arrhythmia and the picture of dilated $\frac{0}{3}$ cardiomyopathy.
Figure 1 Twelve lead surface electrocardiogram (panel $A$ ) and rhythm strip (panel B) obtained on initial visit. The 12 lead electrocardiogram $(A)$ shows a wide $Q R S$ tachycardia with a right bundle branch block and left superior axis morphology and a cycle length of $450 \mathrm{~ms}$. Rhythm strip (right precordial leads) (B) recorded at the other time clearly shows that ventriculoatrial conduction times during the tachycardia progressively lengthen (arrows indicate retrogradely conducted atrial activities) until ventricular impulse fails to conduct to the atrio retrogradely $(5: 4,3: 2,4: 3$ Wenckebach block), clarifying the diagnosis of ventricular tachycardia.

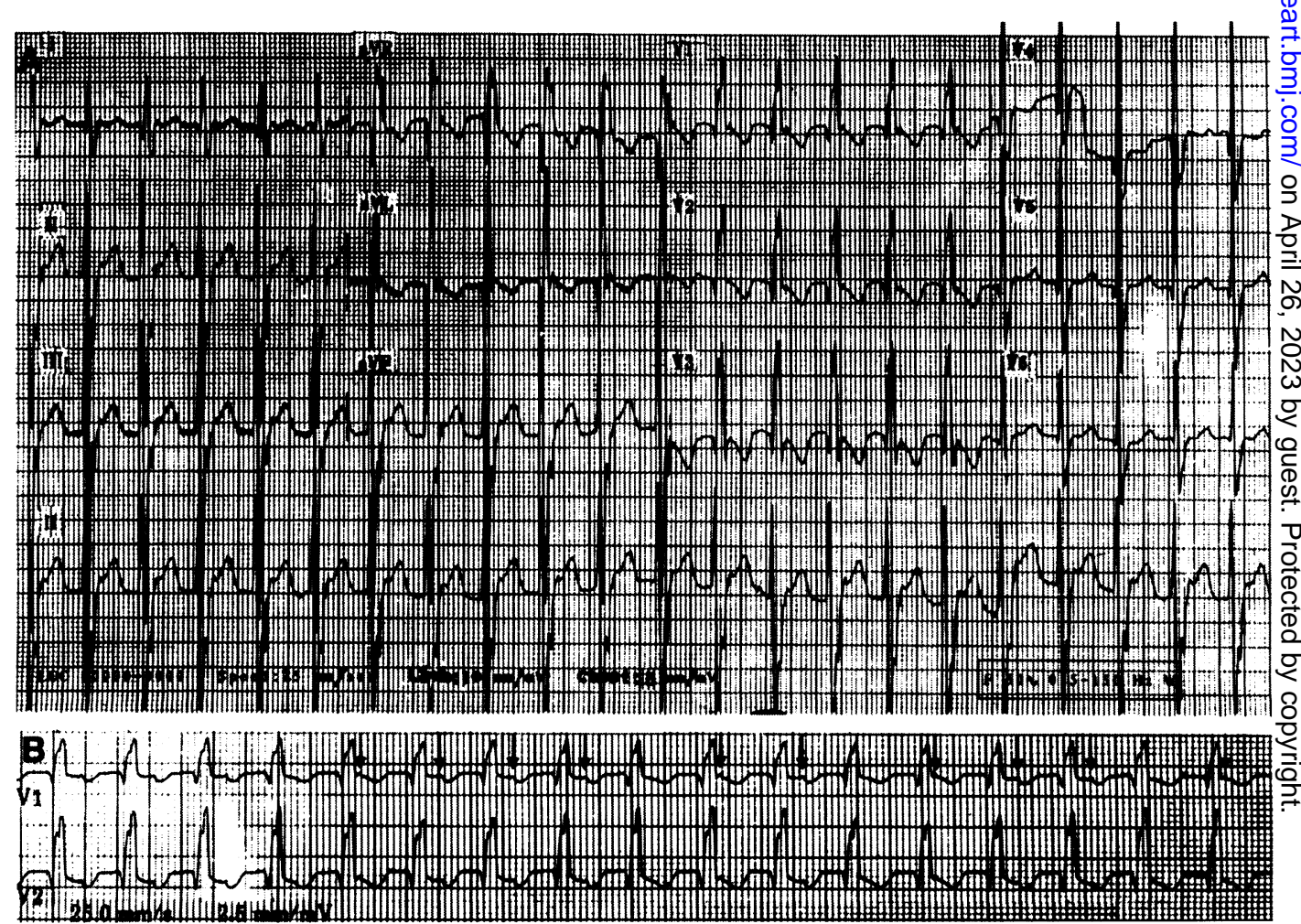


Figure 2 mode echocardiograms obtained on admission (panel $A$ ) and $4 \frac{1}{2}$ months after successful antiarrhythmic treatment with oral verapamil (panel B). The echocardiogram taken during ventricular tachycardia on admission (A) shows severe cardiac dilatation and poor contractility with a shortening fraction of $11 \%$, but follow up examination during sinus rhythm (B) shows normalisation of cardiac dimensions and contractility with $a$ shortening fraction of $34 \%$. $L V E D D$, left ventricular end diastolic dimension; LVESD, left ventricular end systolic dimension.
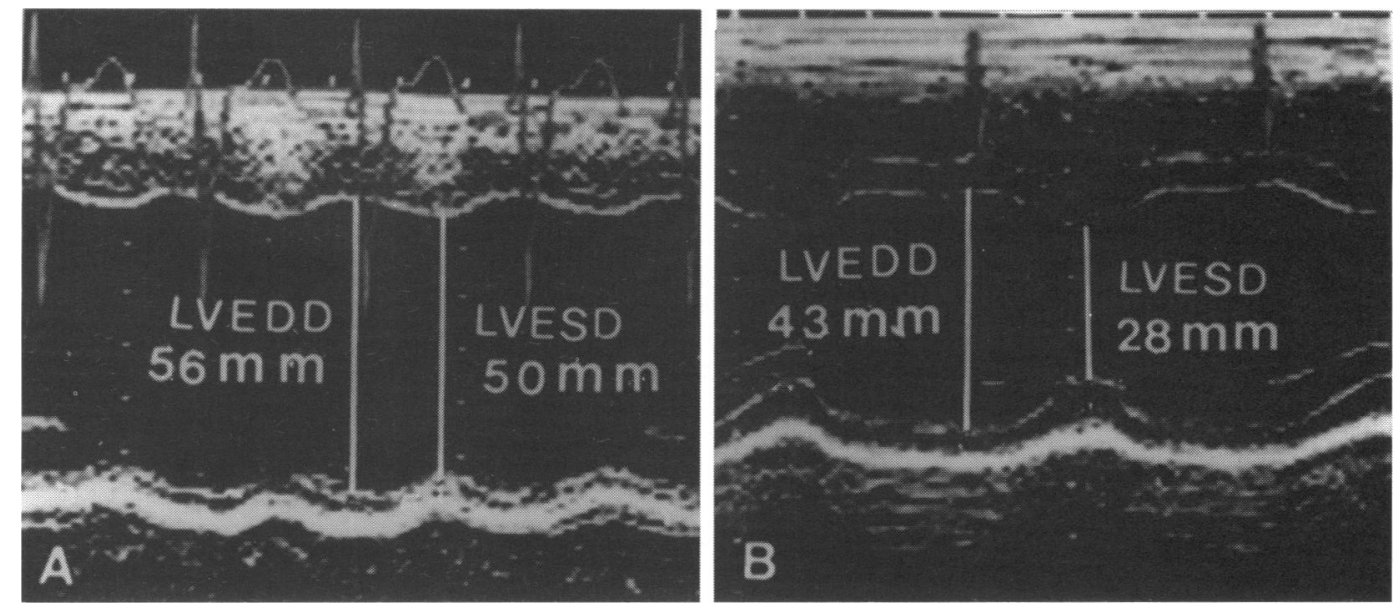

\section{Case report}

A four year old boy was referred to our institution because of a heart murmur and cardiomegaly. On admission, he had signs and symptoms of heart failure, a grade $2 / 6$ systolic murmur at the left lower sternal border and apex, cardiomegaly on chest $x$ ray, and a heart rate of 126 beats/min. The electrocardiogram revealed a wide complex tachycardia with a QRS pattern of right bundle branch block and left axis deviation (QRS axis $-78^{\circ}$ ) at a rate of 125 to 136 beats/min (fig $1 \mathrm{~A}$ ), which showed second degree ventriculoatrial block with varying retrograde Wenckebach periodicity, clarifying the diagnosis of ventricular tachycardia (fig 1B). The Holter recording showed incessant monomorphic ventricular tachycardia. Echocardiographic examination during ventricular tachycardia revealed a dilated hypocontractile left ventricle with a shortening fraction of $11 \%$ (fig $2 \mathrm{~A}$ ), moderate mitral regurgitation, and mild tricuspid regurgitation. Intravenous lignocaine, adenosine, and esmolol could not stop the tachycardia. The patient was treated for both heart failure and tachycardia with diuretics and oral amiodarone for 20 days; however, the tachycardia remained incessant so amiodarone was discontinued. We decided to attempt oral verapamil treatment because the patient had characteristic electrocardiographic findings of verapamil responsive left ventricular tachycardia. Following oral administration of verapamil, $3 \mathrm{mg} / \mathrm{kg} /$ day, normal sinus rhythm dominated in Holter monitoring. Verapamil was gradually increased up to $5 \mathrm{mg} / \mathrm{kg} /$ day. Thereafter, no episodes of ventricular tachycardia were seen on the subsequent Holter recordings, and the child's clinical status improved remarkably. The signal averaged electrocardiogram showed no late potentials. The treadmill exercise test showed no ventricular extrasystoles or ventricular tachycardia. On chest $x$ ray cardiomegaly had disappeared and left ventricular function had gradually normalised (fig 2B). With oral verapamil treatment, the patient has remained asymptomatic, with normal left ventricular function and no recurrence of the ventricular tachycardia over a follow up of 10 months.

\section{Discussion}

In young children with incessant ventricular tachycardia and advanced ventricular dysfunction, treatment of the tachycardia with conventional antiarrhythmic drugs remains a major therapeutic challenge because most of these drugs can cause further depression of myocardial function. Thus cryoablation or surgical resection of the focus has been recommended in those patients. ${ }^{3-5}$ Although there have been a few reports describing the effective use of oral verapamil for chronic idiopathic ventricular tachycardia associated with ventricular dysfunction in adult patients, ${ }^{67}$ such cases have not been described in very young children. Ohe et al reported that the patients with verapamil sensitive severe left ventricular tachycardia were not helped by oral verapamil administration, and all eventually required non-pharmacological treatment, such as catheter ablation, map guided cryosurgery, or an antitachycardia pacing device. ${ }^{2}$

The experience with our patient shows that despite the associated severe ventricular dysfunction, oral verapamil can be effective and safe for acute termination and chronic prophylaxis of the arrhythmia in young children with verapamil responsive ventricular tachycardia. However, further studies are needed to assess the long term efficacy and safety of oral verapamil in this setting.

We are grateful to Dr C Y Cho, Dr J S Kim, and Dr C J Kim for their participation in studying this patient.

1 Lin FC, Finley CD, Rahimtoola SH, Wu D. Idiopathic paroxysmal ventricular tachycardia with a QRS pattern of right bundle branch block and left axis deviation: a unique clinical entity with specific properties. Am f Cardiol 1983; 52:95-100.

2 Ohe T, Aihara N, Kamakura S, Kurita T, Shimizu W, sustained left ventricular tachycardia in patients without structural heart disease. 7 Am Coll Cardiol 1995;25:54-8.

3 Zeigler VL, Gillette PC, Crawford FA, Wiles HB, Fyfe DA New approaches to treatment of incessant ventricular tachycardia in the very young. $f \mathrm{Am}$ Coll Cardiol 1990; 16:681-5.

4 Garson A, Gillette PC, Titus JL, Hawkins E, Kearney D, Ott $\mathrm{D}$, et al. Surgical treatment of ventricular tachycardia in infants. N Engl $¥$ Med 1984;310:1443-5.

5 Fyfe DA, Gillette PC, Crawford FA, Kline CH. Resolution of dilated cardiomyopathy after surgical ablation of ventricular tachycardia in a child. $¥ \mathrm{Am}$ Coll Cardiol 1987;9:231-4.

6 Toivonen L, Nieminen M. Persistent ventricular tachycardia resulting in left ventricular dilatation treated with veraresulting in left ventricular dilata

7 Perez Y, Lellouche D, Nghiem DK, Cachin JC, Castaigne A. Chronic irregular idiopathic ventricular tachycardia with adult. Eur Heart $\mathcal{f}$ 1994;15:1419-22. Shimomura K. Long-term outcome of verapamil-sensitive myocardial dysfunction suppressed by verapamil in an 